\title{
Application of Radial Basis Function Neural Network in Diagnosis of Ms Disease
}

\author{
Mohammad Esmaeil Shaabani ${ }^{1}$, Touraj Banirostam ${ }^{2}$, Alireza Hedayati ${ }^{3}$ \\ Department of Computer Engineering, Tehran Center Branch, Islamic Azad University, Tehran, Iran 1,2,3
}

\begin{abstract}
Artificial intelligence consist of research in computer science that it's targets is creating a computer that could reason such as human and help them make decision better. Decision support system is computer software that design for diagnosis system. Multiple Sclerosis is a kind of disease that starts with damage the myelin of brain and has some neurological symptoms like reducing the power of vision, spastic paralysis of limbs and impotency. In this case, the major problem is the lack of diagnosis. To improve diagnosis, Radial Basis Function Neural Network is used. In this study, we use learning power of Neural Network. K-fold cross validation has been used for optimizing the input/output. Implementation has been done in MATLAB. The Dataset consist of 600 patients that each one has 6 columns, 5 of them is input and one a of them is output that shows diagnosis. Proposed Method RBF compared with ANFIS and MLP. The result shows that RBF's accuracy is $96 \%$. Its accuracy is a little more than ANFIS but it's training time is much more that the other. ANFIS's accuracy is almost $96 \%$. By consideration of too much training time of RBF and ignore the slightly different accuracy, ANFIS give us the best result.
\end{abstract}

Keywords: Multiple Sclerosis, Fuzzy System, Neural Network, Radial Basis Function.

\section{INTRODUCTION}

Medical diagnosis is often done by expertise and experience of physician but sometimes may lead to misdiagnosis. Multiple sclerosis (MS) is a disease of the central nervous system. In it, patient's body produces antibodies that attack and damage the Myelin, in which the myelin sheath (the insulation for nerve fibers) is in trouble and the damage to myelin in the central nervous system cause to disconnect between brain and other organs. NNs are a set of powerful mathematical tools that simulate the way that the human brain deals with information and the procedure of learning. NNs have the ability to identify and learn highly complex and nonlinear relationships from input-output data only, without the use of first principle equations describing the system. This is accomplished by storing information in computational nodes called neurons. The development of an NN is based on a set of inputoutput examples, which the network learns during the training phase. In this stage, the network parameters associated with the neurons and/or the interconnection links are determined using an optimization procedure, which minimizes the errors between the true outputs and the network predictions over a set of training examples. A successfully trained NN model should be capable of producing accurate estimations given a new set of input data. Depending on the way the nodes of the network are interconnected and the calculations that each node performs, NNs are categorized to a variety of architectures [1]. Radial basis function (RBF) neural network is based on supervised learning. RBF networks are also good at modelling nonlinear data and can be trained in one stage rather than using an iterative process and also learn the given application quickly. They are useful in solving problems where the input data are corrupted with additive noise. The transformation functions used are based on a
Gaussian distribution. If the error of the network is minimized appropriately, it will produce outputs that sum to unity, which will represent a probability for the outputs. Radial basis function (RBF) networks [1] constitute a special network architecture that presents some remarkable advantages over other NN types including: (a) better approximation capabilities when performing Interpolation, i.e. providing predictions in-between the training data points, (b) simpler network structures comprising a single hidden layer and (c) faster learning algorithms which are usually split into two stages [2-3]. Due to these advantages, RBF networks have been used extensively for modeling complex systems, with many successful applications in developing medical diagnostic tools [4-5]. Especially in this area, improving the accuracy of the network predictions is vital in achieving high performance diagnosis. In absence of expert and with information that obtained from White Matter Lesions in MRI, early diagnosis of MS is so important. The proposed system can be used in some cases such as lack of access to specialist, help the physicians understand the patient condition. Unfortunately, in many cases in the past most of MS patient couldn't treat their illness, because there was no decision support system. Consultant power to physicians and specialist is the importance of proposed system.

\section{MULTIPLE SCLEROSIS}

This chronic disease starts with damage the myelin of brain and spinal cord that lead to nervous symptoms. Multiple Sclerosis is an unpredictable clinical period that had been detected in white women more [6]. It starts in 20 to 40 years old person but it may happen in any age. It 
starts when white blood cell attack and damage the recognition such as show the damaged area. MRI show the myelin. Myelin is a sheath for nerve fibers and help to waste related to injured myelin in brain with defined limit. transfer message faster. Each time white blood cells attack It shows the lesions that indicate the injured part of tissue nerve fibers relate to the organs, they make problem for and help the physicians in recognition of MS. White that organs [7]. In the case of outbreak MS no specific matter lesion is a kind of structural changes in brain that cause has been found, but Genetic and environmental it's important feature is the destructed myelin of brain. factors have an effect on it. Genetic factors can be noted as MRI indicates this area in white color [11].

Much evidence such as a high incidence of MS have been seen in twins Monozygotic or higher incidence of MS have been seen in family of patients with MS. Among the environmental factors can point to the role of vitamin D3. Physicians believe that shortage of this vitamin has an important role in causing MS. Also among the environmental factors, viral infections like EBV virus is raised. It seems that viruses play an important role in stimulating the immune system and activation of the system against the specific areas of the myelin tissue central system. Important point is that MS disease in genetically susceptible individuals by factors such as lack of vitamin D, smoking and viral infections can occur [8]. Physicians are suspicious to MS when they face the patient with movement and sensorial symptoms. Some of neurological symptoms that seen in MS is seeing blurry sensorial symptoms, lack of balance, spoken failure, paresis and sensory disorders. Physicians diagnose it according to clinical result and diagnostic criteria that could be seen in MRI [8]. The best way to illustrate the points of the central nervous system (brain and spinal cord) in which myelin was destroyed is MRI plaque.

MS detection is done on the basis of plaque in the central nervous system Plaques are seen in cerebral ventricles [6]. Of course, eye scans is an effective factor in detection of MS. Also physicians may request some test on nerves and senses such as vision, hearing and so on. In these experiments, the nerves are stimulated and electrical message transmission speed are measured. Patients with MS have slower response to stimulate of nerves. Physicians may request CFS examination that its fluid is taken from the lower back and tests will be performed on. CSF in patient with MS contain high levels of protein and white blood cells is higher than usual.

\section{III.MAGNETIC RESONANCE IMAGING}

MRI due to high accuracy in diagnosis of soft tissues and is an important tools in diagnosis of diseases because of showing the differentiation of normal tissue and abnormal one, especially disease of the central nervous system. There are many neurological disorders that imply serious damage to the central nervous system among the most common disorders of this type could cite MS disease [10]. MRI has important role in assessment the waste of brain and other tissues to evaluate the disease and related path physiology perception. The best way to show the part of nervous system where myelin is destroyed is MS plaques that could be seen in MRI [8]. The recognition of MS is based on existence of plaques in central nervous system. Plaques are seen around cerebral ventricles. MRI of brain and spinal cord are the most sensitive method of MS

\section{IV.LITERATURE REVIEW}

Ziya Ekşi et al [12] proposed a computer-aided detection system to diagnose MS lesions on FLAIR MR Image. The proposed system uses Fuzzy-C Means (FCM) and morphological operations for segmentation of MS lesions. In their study, the lesions detected by aid of physicians and the lesions detected by means of the proposed system have been compared according to the Jaccard index. Similarity rate in the comparison operation on a total of $90 \mathrm{MR}$ images is calculated as 91.2\%. Ali Amooji and Neda Esmaeilpour Mianji [13] proposed a system that help doctors and patients in providing decision support system, interactive training tools and expert skills. The system constitutes part of intelligent system for diagnosis of neurological diseases that used in one of the great hospital in Tehran. All of the neurological diseases diagnosis have been investigated in this project. The system constitutes part of intelligent system of diagnosis of neurological diseases. The present expert system is evolving and increasing efficiency for all neurological diseases.

Therefore the work was aimed to design a system for the diagnosis of Neurological diseases using FC (Fuzzy Cognitive) which is, a successful application of Lotfizadeh's fuzzy set theory. Usefulness and power of a FC depends on its knowledge base which consists of a data base and a rule base. It is observed that the performance of a FC mainly depends on its rule base, and optimizing the membership function distributions stored in the data base is a fine tuning process. Borgohain and Sanyal [14] argued about the implementation of a rule based expert system for diagnosing neuromuscular diseases. Their proposed system is implemented as a rule based expert system in JESS for the diagnosis of Cerebral Palsy, Multiple Sclerosis, Muscular Dystrophy and Parkinson's disease. In it, the user is presented with a list of questionnaires about the symptoms of the patients based on which the disease of the patient is diagnosed and possible treatment is suggested. The system can aid and support the patients suffering from neuromuscular diseases to get an idea of their disease and possible treatment for the disease. The system is a rule based expert system implemented using the Java Expert System Shell using the backward chaining mechanism. Agboizebeta and Chukwuyeni [15] present a diagnostic fuzzy cluster means system to help in diagnosis of Multiple sclerosis using a set of symptoms. Fuzzy Cluster Means (FCM or Fuzzy CMean) analysis to the diagnosis of different forms of multiple sclerosis is the focal point. Application of cluster analysis involves a sequence of methodological and analytical decision steps that enhances the quality and 
Vol. 5, Issue 7, July 2016

meaning of the clusters produced. This advanced system which uses clustered data set is more precise than the traditional system. The classification, verification and matching of symptoms to the two groups of clusters (Relapsing/remitting multiple sclerosis and Primary Progressive Multiple Sclerosis) was necessary especially in some complex scenarios. Rahman et al. [16] used classic controller and control of nano-robots to identify the damaged parts. Their idea for development of nano-robot is using NN to identify and fix the damaged neurons.

Their system were going to address a disease called demyelination and proposed a nano robotic control system for cure.Datta and Narayana[17] integrated the brain anatomical knowledge with non-parametric and parametric statistical classifiers for automatically classifying tissues and lesions on high resolution multichannel threedimensional images acquired on 60 MS brains. They had presented and implemented a comprehensive and fully automated technique for classifying 3D MR brain images in MS. The proposed automated segmentation technique was assessed quantitatively for THWL classification with the use of quantitative metrics, Bland-Altman and regression analyses.

The proposed technique should allow accurate estimation of the GM and WM atrophy that is shown to correlate with clinical disability in MS. The results of automatic lesion segmentation were reviewed by the expert. The agreement between results obtained by the automated analysis and the expert was excellent as assessed by the quantitative metrics, low absolute volume difference percent (36.18 \pm 34.90$)$, low average symmetric surface distance $(1.64 \mathrm{~mm} \pm 1.30 \mathrm{~mm})$, high true positive rate $(84.75 \pm 12.69)$, and low false positive rate $(34.10 \pm 16.00)$.

The segmented results were also in close agreement with the corrected results as assessed by Bland-Altman and regression analyses. Arabzadeh Ghahazi et al [18] introduced a novel methodology for the segmentation of brain multiple sclerosis (MS) lesions in magnetic resonance imaging (MRI) volumes using a new clustering algorithm named spatially constrained possible fuzzy C-means (SCPFCM). SCPFCM uses membership, typicality, and spatial information to cluster each voxel.

Their proposed method relies on an initial segmentation of MS lesions in $\mathrm{T} 1-\mathrm{w}$ and $\mathrm{T} 2{ }^{-} \mathrm{w}$ images by applying SCPFCM algorithm, and the T1 image was then used as a mask and is compared with $\mathrm{T} 2$ image. Their proposed method was applied to 10 clinical MRI datasets. The results obtained on different types of lesions had been evaluated by comparison with manual segmentations.

They presented a new method to extract MS lesions based on MPFCM algorithm, tested on two sets of simulated and real images. As the results, their method worked better and more accurate by increasing noise. And also in real images, it is better than the other previous ones in extracting tiny lesions.

\section{RADIAL BASIS FUNCTION (RBF)}

An artificial neural network (ANN) has three layers: input layer, hidden layer and output layer. Choice of the number of the hidden layers, hidden nodes and type of activation function plays an important role in model constructions RBFNN can be described as a three-layer feed forward structure as shown in fig.1. It consists of three layers: input layer, hidden layer and output layer. The input layer does not process the information; it only distributes the input vectors to the hidden layer. The hidden layer consists of a number of RBF units $\left(n_{h}\right)$ and bias $\left(b_{k}\right)$.

Each hidden layer unit represents a single radial basis function, with associated center position and width. Each neuron on the hidden layer employs a radial basis function as nonlinear transfer function to operate on the input data. The most often used RBF is Gaussian function that is characterized by a center $\left(c_{j}\right)$ and width $\left(r_{j}\right) \cdot \mathrm{RBF}$ functions by measuring the Euclidean distance between input vector $(\mathrm{x})$ and the radial basis function center $\left(c_{j}\right)$ and performs the nonlinear transformation with RBF in the hidden layer as given in (1):

$$
h_{j}(x)=\exp \left(-\left\|x-c_{j}\right\|^{2} / r_{j}^{2}\right)
$$

In which, $h_{j}$ is the notation for the output of the $\mathrm{jth}$

$\mathrm{RBF}$ unit. For the jth RBF $c_{j}$ and $r_{j}$ are the center and width, respectively. The operation of the output layer is linear, which is given in (2):

$$
y_{k}(x)=\sum_{j=1}^{n h} w_{k j} h_{j}(x)+b_{k}
$$

Where $y_{k}$ is the kth output unit for the input vector $\mathrm{x}$, $w_{k j}$ the weight connection between the kth output unit and the jth hidden layer unit and $b_{k}$ the bias. From (1) and (2), one can see that designing a RBFNNs involves selecting centers, number of hidden layer units, width and weights. There are various ways for selecting the centers, such as random subset selection, K-means clustering, orthogonal least-squares learning algorithm, RBFPLS, etc.

The widths of the radial basis function networks can either be chosen same for all the units or can be chosen different for each unit. In this paper, considerations were limited to the Gaussian functions with a constant width (the same for all units). Forward subset selection routine was used to select the centers from training set samples.

The adjustment of the connection weight between hidden layer and output layer is performed using a least-squares solution after the selection of centers and width of radial basis functions. 


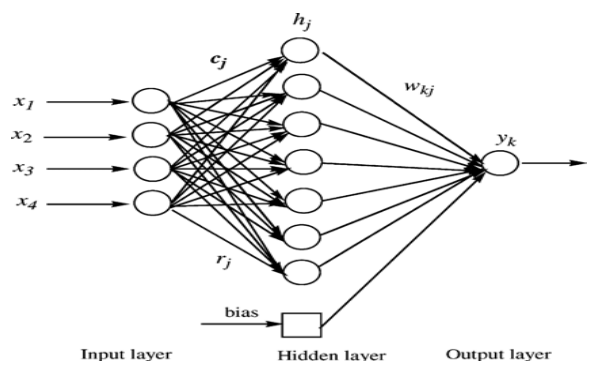

Fig. 1 A typical architecture of RBFNs

\section{VI.TRAINING OF RBF}

A training set is an m labelled pair $\{\mathrm{Xi}, \mathrm{di}\}$ that represents associations of a given mapping or samples of a continuous multivariate function. The sum of squared error criterion function can be considered as an error function $\mathrm{E}$ to be minimized over the given training set. That is, to develop a training method that minimizes $\mathrm{E}$ by adaptively updating the free parameters of the RBF network. These parameters are the receptive field centers $\mu_{j}$ of the hidden layer Gaussian units, the receptive field weights $\sigma_{j}$, and the output layer weights $\left(w_{i j}\right)$. Because of the differentiable nature of the RBF network transfer characteristics, one of the training methods considered here was a fully supervised gradient-descent method. In particular, $\mu_{j}, \sigma_{j}$ and $w_{i j}$ are updated as follows:

$$
\begin{aligned}
& \Delta \mu_{j}=-\rho_{\mu} \nabla_{\mu j} E \\
& \Delta \sigma_{j}=-\rho_{\sigma} \frac{\partial E}{\partial \sigma_{j}} \\
& \Delta w_{i j}=-\rho_{w} \frac{\partial E}{\partial w_{i j}}
\end{aligned}
$$

Where $\rho_{\mu}, \rho_{\sigma}$ and $\rho_{w}$ are small positive constants. This method is capable of matching or exceeding the performance of neural networks with back-propagation algorithm, but gives training comparable with those of sigmoidal type of FFNN. The training of the RBF network is radically different from the classical training of standard FFNNs. In this case, there is no changing of weights with the use of the gradient method aimed at function minimization. In RBF networks with the chosen type of radial basis function, training resolves itself into selecting the centers and dimensions of the functions and calculating the weights of the output neuron.

The center, distance scale and precise shape of the radial function are parameters of the model, all fixed if it is linear. Selection of the centers can be understood as defining the optimal number of base functions and choosing the elements of the training set used in the solution. It was done according to the method of forward selection. Heuristic operation on a given defined training set starts from an empty subset of the base functions. Then the empty subset is filled with succeeding basis functions with their centers marked by the location of elements of the training set; which generally decreases the sumsquared error or the cost function. In this way, a model of the network constructed each time is being completed by the best element. Construction of the network is continued till the criterion demonstrating the quality of the model is fulfilled. The most commonly used method for estimating generalization error is the cross-validation error.

\section{SIMULATION PROPOSED METHOD}

Radial basis function (RBF) neural network is based on supervised learning. RBF networks were independently proposed by many researchers 5-9 and are a popular alternative to the MLP. RBF networks are also good at modelling nonlinear data and can be trained in one stage rather than using an iterative process as in MLP and also learn the given application quickly. They are useful in solving problems where the input data are corrupted with additive noise.

The transformation functions used are based on a Gaussian distribution. If the error of the network is minimized appropriately, it will produce outputs that sum to unity, which will represent a probability for the outputs. The objective of this article is to study the applicability of RBF to diagnose of MS and compare the results with MLP and ANFIS with hybrid learning.

\section{INPUT DATA}

To achieve dataset of patients we use article 15 and society for the protection of MS patients. We use features of white matter forms as follow: volume, Sphericity, compactness, tissue contrast, Environment. The lesion volume, given in terms of the number of voxels and calculated in centimeter. Sphericity evaluates how spherical a lesion is. The more elongated the lesion is and deviates from a sphere, the lower sphericity will be. Compactness evaluates how compact a lesion is. The measure of discrete compactness for a 3D object (Comp3D) relates the enclosing surface area, composed of the neighboring voxels of the object, with its volume. Where the volume is large or the enclosing surface is small, means that the object is strongly compact. Tissue contrast evaluates the minimum color contrast to detect a WML in QMCI images.

The measure of surrounding WM (SWMp) is calculated as a ratio between the surrounding WM and the number of voxels in neighboring the lesion and it is expressed as a percentage value. Essentially, it is defined as the ratio between the volume $\mathrm{V}$ of the lesion and the volume of the smallest sphere enclosing the.

Dataset consist of 600 patients that has 6 columns. 5 of them are white matter feature and 1 of them is result that characterized by 1 (indicate the person has MS) or 2(the person does not have MS).Each row corresponds to a person's features. 


\section{IX.RBF IN DIAGNOSIS SYSTEM}

The RBF neural network architecture considered for this application was a single hidden layer with Gaussian RBF. The basis function $\phi$ is a real function of the distance (radius) $\mathrm{r}$ from the origin, and the center is $\mathrm{c}$. The most common choice of $\phi$ includes thin-plate spline, Gaussian and multi quadric. Gaussian-type RBF was chosen here due to its similarity with the Euclidean distance and also since it gives better smoothing and interpolation properties. The choice of nonlinear function is not usually a major factor in network performance, unless there is an inherent special symmetry in the problem.

Training of the RBF neural network involved two critical processes. First, the centers of each of the J Gaussian basis functions were fixed to represent the density function of the input space using a dynamic ' $\mathrm{k}$ means clustering algorithm'. This was accomplished by first initializing the set of Gaussian centers $\mu_{j}$ to random values. Then, for any arbitrary input vector $X^{(t)}$ in the training set, the closest Gaussian center, $\mu_{j}$, is modified as:

$$
\mu_{j}^{\text {new }}=\mu_{j}^{\text {old }}+\alpha\left(x^{(t)}-\mu_{j}^{\text {old }}\right)
$$

Where $\alpha$ is a learning rate that decreases over time. This phase of RBF network training places the weights of the radial basis function units in only those regions of the input space where significant data are present. The parameter $\sigma_{j}$ is set for each Gaussian unit to equal the average distance to the two closest neighbour Gaussian basis units. If $\mu_{1}$ and $\mu_{2}$ represent the two closest weight centers to Gaussian unit $\mathrm{j}$, the intention was to size this parameter so that there were no gaps between basis functions and only minimal overlap between adjacent basis functions were allowed.

After the Gaussian basis centers were fixed, the second step of the RBF network training process was to determine the weight vector $\mathrm{W}$ which would best approximate the limited sample data $\mathrm{X}$, thus leading to a linear optimization problem that could be solved by ordinary least squares method. This avoids the problem of gradient descent methods and local minima characteristic of back propagation algorithm. For MLP network architecture, a single hidden layer with sigmoid activation function, which is optimal for the dichotomous outcome, is chosen. A back propagation algorithm based on conjugate gradient optimization technique was used to model MLP for the above data.

\section{SIMULATION RESULT}

We had implemented the proposed method RBF and compare it with Anfis with hybrid learning and MLP. We use Confusion matrix to evaluate result and compare the methods:
TP: How many MS patients are diagnosed correctly.

FP: How many MS patients were diagnosis wrong as healthy.

TN: How many healthy person were diagnosed correctly as healthy.

FN: How many healthy people were diagnosed wrong as MS patient.

P: How many people were calssified correctly.

$\mathrm{N}$ : How many people are classified incorrectly.

Sensitivity: How much this test could select MS patient correctly.

$$
T P R=T P / P=T P /(T P+F N)
$$

Specificity: It means the ability of test to select the healthy person.

$$
S P C=T N / N=T N /(F P+T N)
$$

Accuracy: availability of system in diagnosis and classify the cases.

$$
A C C=(T P+T N) /(P+N)
$$

We used dataset in ANFIS and MLP and compare it with proposed RBF. We used them in same situation and use Kfold cross validation in all of them and the result are:

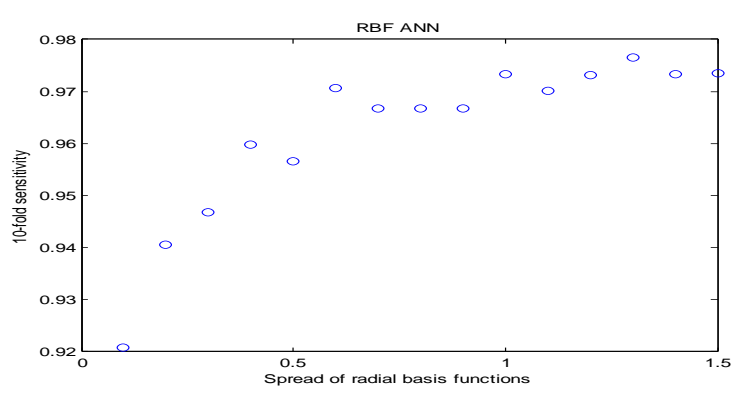

Fig. 2. sensitivity in RBF

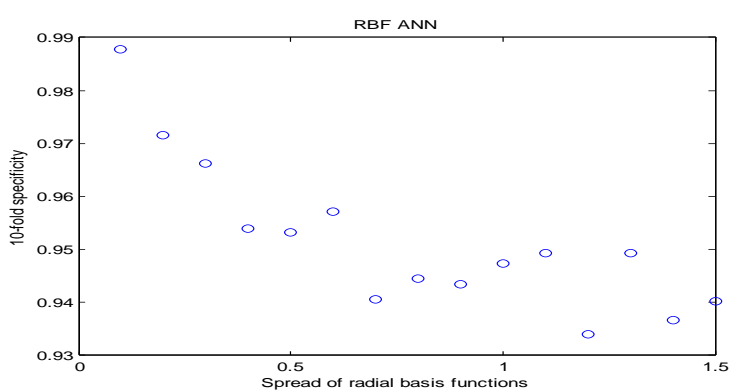

Fig. 3 Specificity in RBF

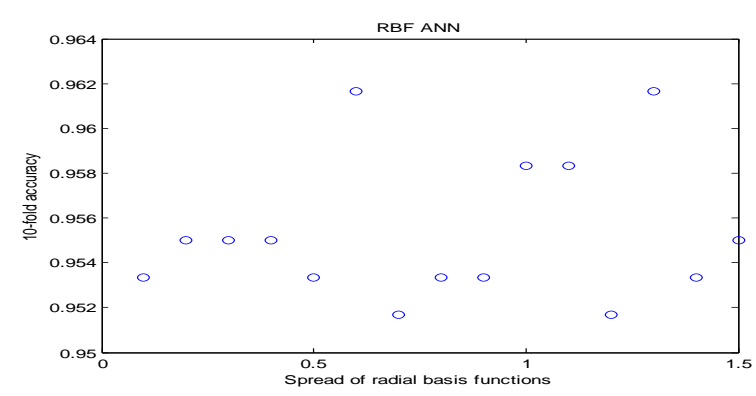

Fig. 4 Accuracy in RBF 


$$
\begin{gathered}
T P R=28.5 /(28.5+0.9)=0.9693 \\
S P C=29.2 /(1.4+29.2)=0.9542 \\
A C C=(28.5+29.2) /(28.5+0.9+1.4+29.2)=0.9616
\end{gathered}
$$

Vol. 5, Issue 7, July 2016

The result of simulation show that RBF has 96\% sensitivity (select MS patients correctly) and 95\% specificity (select healthy person) and has $96 \%$ accuracy in diagnosis.

In simulation with MLP the result as follow:

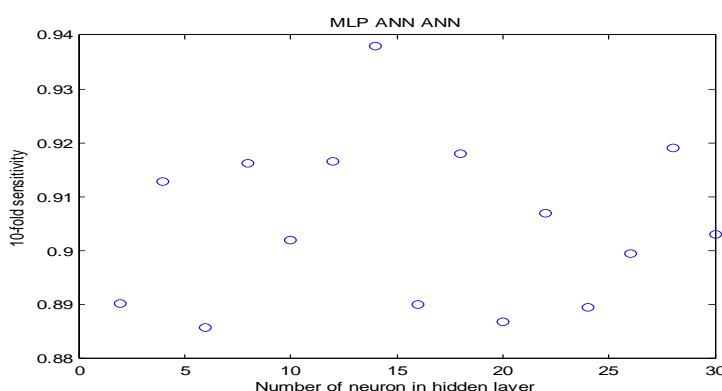

Fig. 5 Sensitivity in MLP

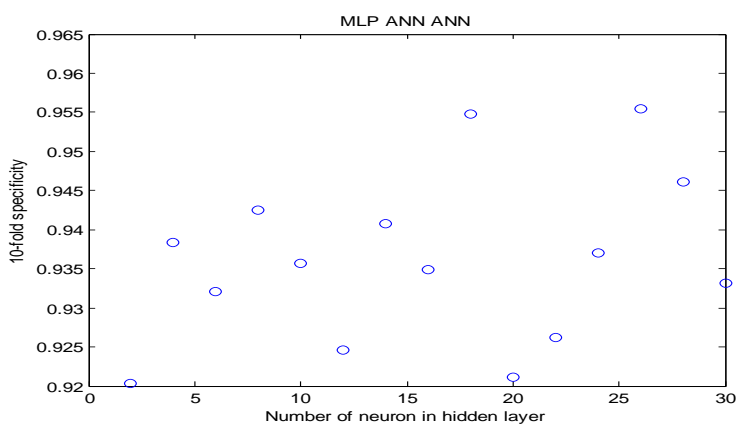

Fig. 6 Specificity in MLP

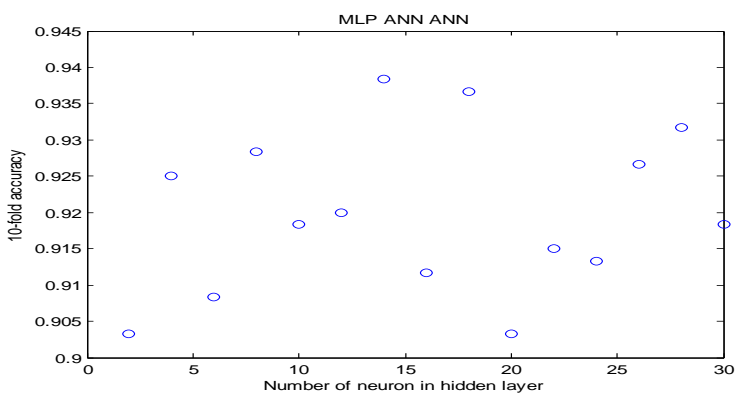

Fig. 7 Accuracy in MLP

$$
\begin{gathered}
\text { TPR }=27.5 /(27.5+1.9)=0.9353 \\
\text { SPC }=28.8 /(28.8+1.8)=0.9411 \\
A C C=(27.5+28.8) /(27.5+1.9+1.8+28.8)=0.9383
\end{gathered}
$$

The result of simulation show that MLP has 93\% sensitivity (select MS patients correctly) and $94 \%$ specificity (select healthy person) and has almost $93 \%$ accuracy in diagnosis.
In ANFIS that use the hybrid learning algorithm the result are as follow:

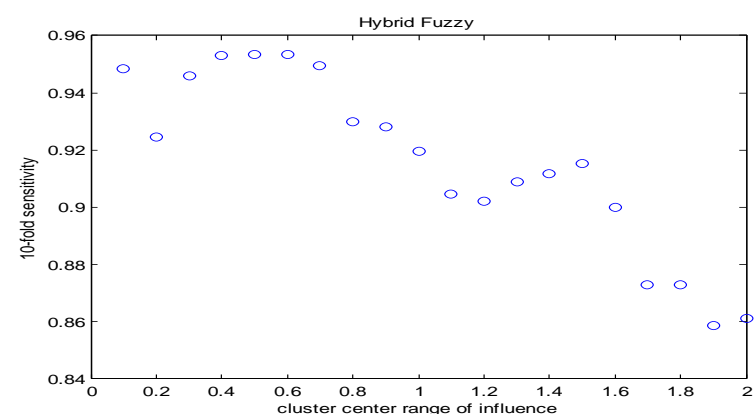

Fig. 8 Sensitivity in ANFIS with hybrid learning

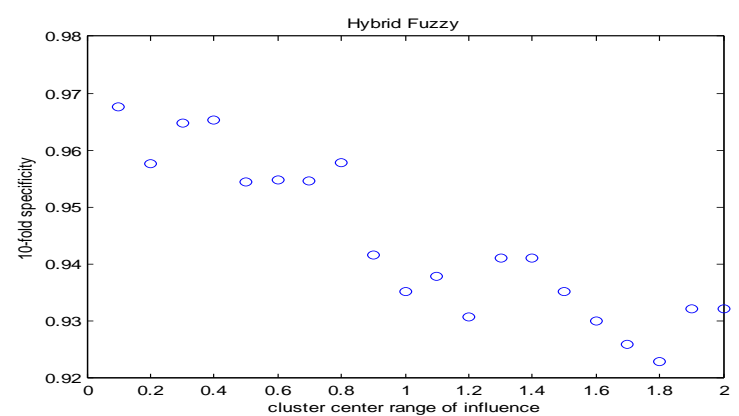

Fig. 9 Specificity in ANFIS with hybrid learning

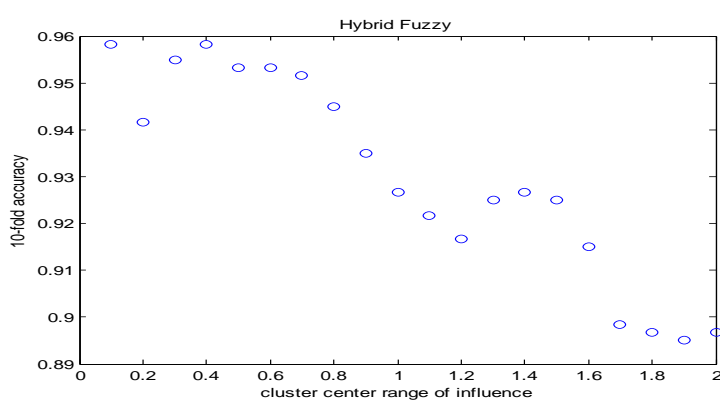

Fig. 10 Accuracy in An fis with hybrid learning

$$
\begin{gathered}
T P R=27.9 /(27.9+1.5)=0.9489 \\
S P C=29.6 /(29.6+1)=0.9673 \\
A C C=(27.9+29.6) /(27.9+1.5+1+29.6)=0.9583
\end{gathered}
$$

The result of simulation show that RBF has 94\% sensitivity (select MS patients correctly) and 96\% specificity (select healthy person) and has almost $96 \%$ accuracy in diagnosis.

\section{XI.CONCLUSION}

Decision support system has an important role in patient care. False detection in each kind of diseases have an irreparable damage to patients and clinican. The aim of this study is improve diagnosis of MS. We had tried to use the learning ability of NN to improve the diagnosis of MS. We use 600 patient's data consist of 5 features. For optimize the input data and evaluate the performance of 


\section{International Journal of Advanced Research in Computer and Communication Engineering}

\section{ISO 3297:2007 Certified}

Vol. 5, Issue 7, July 2016

our system we use K-fold cross validation. Proposed system compared with ANFIS with hybrid learning and MLP. Simulation result shows that RBF has more accuracy than ANFIS and ANFIS has more accuracy than MLP. RBF has more accuracy but it's learning time is so longer than ANFIS. As result show that by consideration of too much training time of RBF and ignore the slightly different accuracy, proposed ANFIS give us the best result.

TABLE 1 Comparison table

\begin{tabular}{|c|c|c|c|}
\hline & specificity & sensitivity & accuracy \\
\hline RBF & 0.9693 & 0.9542 & 0.9616 \\
\hline $\begin{array}{c}\text { ANFIS with hybrid } \\
\text { learning }\end{array}$ & 0.9489 & 0.9673 & 0.9583 \\
\hline MLP & 0.9353 & 0.9411 & 0.9383 \\
\hline
\end{tabular}

\section{REFERENCES}

[1] Ghosh-Dastidar. S, Adeli. H, Dadmehr. N, "Principal component analysisenhanced cosine radial basis function neural network for robust epilepsyand seizure detection.", IEEE Trans Biomed Eng,55,512-518,(2008)

[2] Alexandridis. A, Chondrodima. E and Sarimveis. H, "Radial Basis Function network training using a non-symmetric partition of the input space and Particle Swarm Optimization." IEEE Trans Neural Netw Learn Syst, 24, 219-230, (2013).

[3] Alexandridis. A, Sarimveis. H and Ninos. K, "A radial basis function network training algorithm using a non-symmetric partition of the input space application to a model predictive control configuration", Adv Eng Software ,42,830-837,( 2011).

[4] Sateesh Babu G \& Suresh. S, "Parkinson's disease prediction using gene expression a projection based learning meta-cognitive neural classifier approach" Exp Syst Appl,40,1519-1529,(2013).

[5] Wei $\mathrm{CH}, \mathrm{Li} \mathrm{Y}$ and Huang PJ, "Mammogram retrieval through machine learning within BI-RADS standards." , J Biomed Inform,44, 607-164, (2011).

[6] Soltanzadeh. A, Neurological and muscles disease.(Tehran, Iran.)

[7] Abdullah \& Bassem. A, Segmentation of Multiple Sclerosis Lesions in Brain MRI. The Libraries website(2012).

[8] Tabrizi. N, Eatemadifar. M, Sharifi. E \& Mirmehdi. R, Multiple Sclerosis. (Tehran, ghaed, 2012).

[9] Smelterz. S, brandgi. B, Heinkel. J and chioyer. K, "Neurology", (Tehran, Year: 2010)

[10] Masroor, M. "MS lesions on MRI image segmentation", The 8th Symposium on Advances in Science and Technology (8thSASTech), Mashhad, Iran, (2012).

[11] Abedini. M, Abian. M \& moatamedinasab. F, MS and complementary medicine, (sari, sheflyn, 2012).

[12] Z. Eksi, M.E. Özcan, A. Aralamak, E. Dandil and Murat Çakiroglu, "Automatic Computer-Aided Detection of Multiple Sclerosis (MS) Lesionson MR Images", 19th National Biomedical Engineering Meeting (BIYOMUT), Pages: 1 - 4,(2015).

[13] Ali Amooji \& Neda Esmaeilpour Mianji, "A Fuzzy expert system for diagnosis of Multiple Sclerosis and Brain Tumor diseases", International Research Journal of Applied and Basic Sciences, Vol 9 (11), P:2055-2059,(2015).

[14] Borgohain. R \& Sanyal. S, "Rule Based Expert System for Diagnosis of Neuromuscular Disorders." International Journal of Advanced Networking and pplications, Volume. 34, Issue. 31, pp.1509-1513 (2012).

[15] Agboizebeta. I.A \& Chukwuyeni. O.J, "Cognitive analysis of multiple sclerosis utilizing fuzzy cluster means.", International Journal of Artificial Intelligence \& Applications (IJAIA), Vol. 3, No. 1,( January 2012).

[16] Masudur. S.M, Al-Arif. R, Quader. N, Shaon. A.M \& Khairul. K, "Sensor based Autonomous Medical Nanorobots. A cure to Demyelination." Cyber Journals Multidisciplinary Journals in Science and Technology, Journal of Selected Areas in Nanotechnology (JSAN), Y.2011.
[17] Datta. S \& Narayana. P.A, "A comprehensive approach to the segmentation of multichannel three-dimensional MR brain images in multiple sclerosis." NeuroImage: Clinical, vol.2, Y.2013, pp.184-196.

[18] Arabzadeh Ghahazi. M, Fazel Zarandi. M.H, Rahimi DamirchiDarasi. S \& Harirchian. M.H, "Fuzzy Rule based Expert System for Diagnosis of Multiple Sclerosis." IEEE Conference on Norbert Wiener in the 21 st Century $(21 \mathrm{CW})$, pp. $1-5,(2014)$.

[19] Doual. N, Csaba. H, De Roo. J, Papageorgiouc. E.I \& Jaulent. M.C "Diagnosis Support System based on clinical guidelines: comparison between Case-Based Fuzzy Cognitive Maps and Bayesian Networks." Conference on computer methods and programs in biomedicine, vol.113, Issue.1, pp.133-143,(2014).

[20] Agharezaei. Z, Bahaadinbeigyb. K, Tofighi. Sh, Agharezaei. L \&Nemati,A, "Attitude of Iranian Physicians and Nurses toward a Clinical Decision Support System for Pulmonary Embolism and Deep Vein Thrombosis.", Conference on Computer Methods and Programs in Biomedicine. Vol. 115, pp.95-101,(2014).

[21] Dr.C.Loganathan \& K.V.Girija, "Hybrid Learning For Adaptive Neuro Fuzzy Inference System.", International Journal Of Engineering And Science, Vol.2, Issue 11, pp. 6-13,(2013).

[22] El-Sappagh. S.H \& El-Masri. S, "A distributed clinical decision support system architecture." Journal of King Saud University Computer and Information Sciences, Vol. 26, Issue 1, pp.6978,(2014).

23] M.E. Shaabani, T. Banirostam and A. Hedayati, "Design and Implementasion of Neuro Fuzzy System for Diagnosis of Multiple Sclerosis", IJCSN International Journal of Computer Science and Network, Volume 5, Issue 1, February 2016.

[24] Nguyen. T, Khosravi. A, Creighton. D \& Nahavandi. S, "Medical Diagnosis by Fuzzy Standard Additive Model with Wavelets." IEEE International Conference on Fuzzy Systems (FUZZ-IEEE) Beijing, China, pp. 1937 - 1944,(2014).

[25] Vijay Kumar Garg \& Dr. R.K. Bansal, "Soft Computing Technique Based on ANFIS for the Early Detection of Sleep Disorders." IEEE International Conference on Advances in Computer Engineering \& Applications (ICACEA-2015), pp. 76 - 79,(2015) 